\title{
Cosmetic surgeries as conspicuous consumption: Disclosing information about having undergone cosmetic surgery signals social status
}

\author{
Michał Folwarczny ${ }^{1}$ and Tobias Otterbring ${ }^{2}$ \\ ${ }^{1}$ Department of Business Administration, Reykjavik University \\ ${ }^{2}$ Department of Management, University of Agder and Institute of Retail Economics
}

\begin{abstract}
Despite the risks associated with cosmetic procedures, the global cosmetic surgery market is growing rapidly. What motivates consumers to engage in these costly and risky beautification efforts? The existing literature, laypeople, and plastic surgeons point to variables such as thinking styles, social media use, and aesthetics-related reasons. Drawing on evolutionarybased theories, we challenge these assumptions by proposing and providing empirical evidence for the notion that both men and women can increase their inferred social status by disclosing participation in cosmetic procedures (Studies 1-3). In addition, women—but not men-receive a "beauty premium" and hence are perceived as more physically attractive when they disclose such information, leading to an increase in their perceived social status (Study 4). Finally, we show that status seeking, both measured and manipulated, predicts consumers' willingness to undergo cosmetic procedures (Study 5). Taken together, our results extend previous research by showing that consumers may "go under the knife" to signal social status.
\end{abstract}

Keywords: social status, social rank, physical attractiveness, conspicuous consumption, beautification, cosmetic surgery

You know, let's put it this way, if all the people in Hollywood who have had plastic surgery, if they went on vacation, there wouldn't be a person left in town.

-Michael Jackson

Whereas emergency surgery saves lives in danger, people who voluntarily undergoe cosmetic surgery put their life at risk; therefore, it should be prudent to avoid the latter. Nonetheless, gluteal fat grafting, which has a relatively high mortality rate of 1:6000, is one of the fastest-growing aesthetic procedures, tapping into a lucrative global cosmetic surgery market that is likely to double this decade and reach $\$ 150$ billion by 2030 (Grand View Research, 2022; Mofid et al., 2017). Interestingly, some consumers advertise their involvement in these risky practices. For example, one study found that Twitter users_-potential patients_post ten times

(D) Michał Folwarczny (1D) Tobias Otterbring

Correspondence concerning this article should be addressed to Tobias Otterbring. E-mail: tobias.otterbring@uia.no

The order of authorship was determined by the toss of a coin and should be considered equal. This project was funded by the Aarhus University Research Foundation: AUFF Starting Grant to Tobias Otterbring.

Declaration of Interest: The authors declare no conflict of interest. more content about cosmetic procedures than do plastic surgeons (Branford et al., 2016). What drives consumers to "go under the knife" and advertise this fact?

Consumer choices can be understood by analyzing the proximate and ultimate causes of behavior (Durante \& Griskevicius, 2018; Otterbring, 2021). Whereas the study of proximate causes underlying consumer behavior focuses on how the behavior occurs, the study of ultimate causes focuses on why the behavior occurs; i.e., what adaptive goals the behavior helps to fulfill (Otterbring et al., 2020; Saad, 2013). Most of the previous studies examining the antecedents of involvement in cosmetic procedures have focused on proximate causes such as the desire to improve appearance, social media use, thinking styles, self-concept clarity, and disposable income levels (e.g., Dorneles de Andrade, 2010; Gimlin, 2000; Grand View Research, 2022; J. Wang \& Yu, 2022). These explanations are insufficient, however, as it is still not clear why a person would benefit from, for example, promoting their surgically-straightened nose on social media? The current research investigates the ultimate causes of involvement in cosmetic surgery. Building on the fundamental motives framework developed to group several key motives driving human behavior (Griskevicius \& Kenrick, 2013; Kenrick et al., 2013; Schaller et al., 2017), we propose that signaling participation in cosmetic surgery is a means by which some consumers signal their social status.

Humans strive for status in part because those at the top 
of a social ladder-compared to those at the bottom-have more influence over group decisions, with such decisions often made to their own advantage (Berger et al., 1972). High-status men are more successful on the mating market compared to their low-status counterparts (Buss, 1989), but women also demonstrate their status superiority in mating contexts (Li et al., 2002; Y. Wang \& Griskevicius, 2014). Even in apparently status-irrelevant situations, high-status individuals have survival-related advantages over their lowerstatus peers, such as higher survival rates after a heart failure (Heo et al., 2012). In sum, the pursuit of status is a fundamental human motive found across cultures and social groups, with higher (vs. lower) status being positively associated with well-being and self-esteem as well as mental and physical health (for a review, see Anderson et al., 2015).

Critical for the current research is the fact that consumers enjoy the benefits of their high status not because they possess certain attributes, but because they are perceived as such by others (Driskell \& Mullen, 1990). To be an effective status signal, a behavior must be costly to the actor such that only a few high-status actors should be able to incur costs associated with the given behavior (Zahavi, 1975). Therefore, to signal a high position in the social hierarchy, consumers sometimes take risky and costly actions (Fessler et al., 2014; Griskevicius et al., 2010; Nunes et al., 2011; Y. Wang et al., 2021). For example, conspicuous consumption, defined as the lavish display of possessions, has traditionally been a socially acceptable (albeit economically wasteful) way to signal status because only high-status people have such a wealth of resources that they can invest solely for the purposes associated with signaling - consumers who are lower in social rank do not have sufficient resources to invest in these costly status affirmations (e.g., Gierl \& Huettl, 2010; Griskevicius et al., 2007; Otterbring et al., 2018; Saad \& Vongas, 2009; Veblen, 1899).

Involvement in cosmetic surgery can serve as a status signal for several reasons. First, cosmetic surgeries are physically costly, with patients accepting long recovery times and surgical risks that can sometimes be severe (Mofid et al., 2017). Second, consumers perceive cosmetic surgeries as financially costly (see pilot study). Third, Internet searches for terms related to cosmetic surgery is higher in places with greater status inequality than in places with less status inequality (X. Wang et al., 2021), supporting the notion that cosmetic surgery involvement can be a way to compensate for status threats (cf. Ivanic et al., 2011). Finally, considering that cosmetic procedures aim to make a person more physically attractive, and that physical attractiveness is positively related to inferred social status (Kalick, 1988), it is plausible that disclosing involvement in cosmetic procedures will increase inferred social status through increased physical attractiveness under certain circumstances.

We contacted four US-based plastic surgeons to determine whether they recognized the potential status-enhancing function of involvement in cosmetic surgery. Specifically, we asked them whether patients sought out their clinics primarily to enhance their appearance or, rather, to improve their social status. All but one indicated the former. The remaining surgeon agreed that people visited his clinic primarily for aesthetic reasons, but also mentioned the status-enhancing function of such procedures. Interestingly, one surgeon mentioned that some patients shared their surgical journey on social media. It is plausible, then, that at least some patients take advantage of the potential status-signaling function of cosmetic surgeries.

In what follows, we test three novel propositions: (a) disclosure of information about involvement in cosmetic procedures increases inferred social status (Studies 1-3); (b) for women, but not men, this effect is partially driven by an inferred boost in their physical attractiveness (Study 4); and (c) experimentally inducing a sense of relatively low (vs. high) social status increases consumers' propensity to "go under the knife" (Study 5).

\section{General Method and Overview of Studies}

In a pilot study, we asked people whether they considered cosmetic surgery to be a form of status consumption. Studies 1-4 tested the prediction that disclosing information about undergoing cosmetic surgery would increase status inferences. Study 1 demonstrated the main effect in a US sample, and Study 2 tested the generalizability of this effect in a UK sample, excluding several alternative explanations. In Study 3, we conceptually replicated the earlier results with a different manipulation and a different way of capturing the dependent variable, while also examining whether our effects were moderated by the sex of the person being assessed. In Study 4, we identified a mechanism responsible for the shift in inferred social status (see Figure 1, for an overview of the main results and the distribution of responses). Finally, in Study 5, we tested the causality of our effects-whether the pursuit of status increases the desire for cosmetic surgeryfinding that experimentally induced feelings of lower (vs. higher) status increases (vs. decreases) consumers' willingness to undergo cosmetic surgery.

Materials and data used in this research are available online on the Open Science Framework (OSF) and can be accessed at https : //osf.io/b75ae/?viewonly = b6365154a40c4b6e9935a69cd69d0284. The studies were designed in PsyToolkit (Stoet, 2010, 2017). All participants were recruited through Prolific Academic. To ensure high data quality, we only included participants whose acceptance rate was $99 \%$ and higher and who had not participated in any of our previous experiments. The studies complied with the local regulations for conducting research involving human subjects. 


\section{Pilot Study}

We conducted a pilot study to investigate the common beliefs about what motivates patients to undergo cosmetic surgeries. Qualitative interviews indicate that women undergo such procedures primarily to improve their physical appearance (Dorneles de Andrade, 2010; Gimlin, 2000). We contest this assumption and test whether people consider cosmetic surgeries as a form of status-signaling consumption.

We recruited 100 participants (61 women, $M_{\text {age }}=31.8$ years, $S D=10.4$ ) from the United States. First, they read and accepted a consent form. Then, they chose which statement they agreed with the most out of two existing ones ("People undergo cosmetic surgeries to improve their physical appearance;" "People undergo cosmetic surgeries to increase their social status"). Next, on a scale anchored at 1 $=$ Disagree Strongly and $7=$ Agree Strongly, they indicated their agreement with three statements about cosmetic surgeries ("Cosmetic surgeries are expensive"; "Cosmetic surgeries are a form of luxury consumption"; "Few people undergo cosmetic surgeries").

We found that a significantly larger proportion of people believed that patients choose cosmetic surgery to improve their physical appearance $(87 \%)$ rather than to increase their social status $(13 \%), \chi^{2}(1, N=100)=54.76, p<.001, \phi$ $=.74$. This effect was not moderated by participants' sex, $\chi^{2}(1, N=100)=2.19, p=.138, \phi=.15$. Although $13 \%$ of the participants chose social status as the primary reason for undergoing cosmetic procedures, qualitative data suggested that none of them mentioned social status - their subsequent free-text responses were consistently related to physical appearance.

The sample mean on the item asking participants whether cosmetic surgeries are expensive was higher than the scale midpoint of $4\left(M_{\text {expensive }}=6.41, S D=0.73\right), t(99)=33.19$, $p<.001, d=3.32$, verifying that participants perceive cosmetic surgeries as pricey. Similarly, the sample mean on the item asking participants whether cosmetic surgeries constitute a form of luxury consumption was higher than the scale midpoint $\left(M_{\text {luxury consumption }}=6.22, S D=0.99\right), t(99)=$ $22.41, p<.001, d=2.24$, indicating that such surgery practices are perceived as a form of luxury consumption. However, participants were not particularly prone to think that only a few people underwent cosmetic surgeries, as the sample mean did not differ significantly from the scale midpoint $\left(M_{\text {prevalence }}=3.78, S D=1.51\right), t(99)=-1.46, p=.926, d=$ -0.15 .

\section{Study 1}

Participants in the pilot study believed that people undergo cosmetic procedures primarily for appearance-related reasons, although they agreed that such procedures are both expensive and a form of luxury consumption, even though such practices are considered relatively common among consumers. In Study 1, we test the prediction that disclosure of information about undergoing cosmetic procedures is an effective status signal; that is, a signal leading to an increase in inferred status.

\section{Participants and Procedure}

We estimated the sample size a priori by a stochastic power simulation in R (Bolker, 2007). Previous research has shown that manipulating posts about people's lifestyle can lead to medium-to-large effect sizes in status inferences (Bellezza et al., 2017). Therefore, in the power simulation, we assumed a medium-to-large effect size of Cohen's $d=$ 0.65 . Our simulation indicated that approximately 65 participants per cell would be sufficient to achieve a power of .95 to detect an effect size of this magnitude with an alpha of .05 . Therefore, we recruited 125 participants (61 women, $M_{\text {age }}=$ 32.5 years, $S D=11.4$ ) from the United States.

After agreeing to an informed consent form, participants were assigned to either an experimental $(n=60)$ or a control $(n=65)$ condition. They were instructed to carefully read the information under a series of Instagram screenshots (i.e., the hashtags) associated with a fictional female character. We created two Instagram profiles with images and took screenshots of six posts purportedly belonging to a character named Alicia M. Hills. The posts were merged into one large image. Each post contained three hashtags. The posts did not include information about the profile owner's physical characteristics. Participants in the experimental condition saw a profile showing Alicia's journey from before to after surgery, with hashtags such as "\#nosejob" and "\#recovery." Participants in the control condition viewed a profile showing Alicia's daily activities, such as eating breakfast and coming home, with hashtags such as "\#coffee" and "\#Sunday."

While viewing the Instagram profiles, participants indicated their agreement with three manipulation check items ("She invests a lot of money to look good"; "Beauty is important for her"; "She spends a lot of time to improve her physical appearance") on a scale anchored at $1=$ Strongly Disagree and $7=$ Strongly Agree. We averaged these items to create a manipulation check index $(\alpha=.94, M=4.83$, $S D=1.68$ ), which measured the beautification efforts of the fictional character.

Next, we measured the perceived social status of the fictional character by adapting three established measures of inferred social status (Bellezza et al., 2017). Participants rated Alicia's social status ("On a scale from 1 to 7 , how would you rank the social status of Alicia?") on a scale anchored at $1=$ Low social status and $7=$ High social status; her financial wealth ("Do you think she is financially wealthy?") on a scale anchored at $1=$ Not wealthy and $7=$ Extremely wealthy; and her income level by indicating agreement ("She has a high income level") on a scale anchored at $1=$ Strongly 
disagree and $7=$ Strongly agree. We averaged responses to these items to create a social status index $(\alpha=.91, M=4.78$, $S D=1.26)$. Finally, participants provided demographic information.

\section{Results and Discussion}

\section{Manipulation check}

A Welch two-sample $t$-test on the index of the manipulation check items revealed that participants rated Alicia's beautification efforts as being substantially higher in the experimental condition than in the control condition $\left(M_{\text {experimental }}=6.09, S D=1.12 ; M_{\text {control }}=3.66, S D=1.21\right)$, $t(123.00)=-11.66, p<.001, d=2.08$.

\section{Inferred status}

We performed a similar analysis with the index of social status as the dependent variable. The analysis revealed that participants rated Alicia's social status to be materially higher in the experimental condition than in the control condition $\left(M_{\text {experimental }}=5.60, S D=0.75 ; M_{\text {control }}=4.03, S D\right.$ $=1.15), t(110.91)=-9.11, p<.001, d=1.60$.

\section{Discussion}

Study 1 provides initial evidence supporting our theorizing-participants rated a fictional character who disclosed information about involvement in cosmetic procedures as higher in social status than a fictional character who disclosed information about everyday activities unrelated to such forms of consumption (see Figure 1).

\section{Study 2}

Study 2 was conducted to test the generalizability of our results across populations; therefore, we recruited a sample from another country. Because the experimental stimuli we used mentioned surgeries that might be perceived as aversive stimuli by some participants, the second goal of Study 2 was to control for potential confounding effects resulting from differences in negative affect between conditions. Finally, the control stimuli used in Study 1 might have signaled low social status (e.g., using public transportation or eating fast food for lunch). To rule out this possible confound, we added a second control condition.

\section{Participants and Procedure}

Because we obtained effect sizes in Study 1 that were considerably larger than those we had assumed in the a priori power simulation, we reduced the sample size to 100 participants ( 66 women, $M_{\text {age }}=36.6$ years, $S D=12.5$ ) recruited from the United Kingdom.

After agreeing to an informed consent form, participants were randomly assigned to either an experimental condition $(n=38)$ or one of two control conditions in which they saw photos of the fictional female character from the previous study $(n=37)$ or solely the Instagram $\log 0(n=25$; variability in cell sizes results from random assignment to experimental conditions). To avoid confusion, participants who saw the Instagram logo were informed that they did not see any silhouette on purpose. Next, they answered the manipulation check items described in Study 1 which were averaged to create a manipulation check index $(\alpha=.93, M=4.44$, $S D=1.64)$, followed by the same social status items, also averaged to create a social status index $(\alpha=.82, M=4.49$, $S D=1.08$ ).

\section{Figure 1}
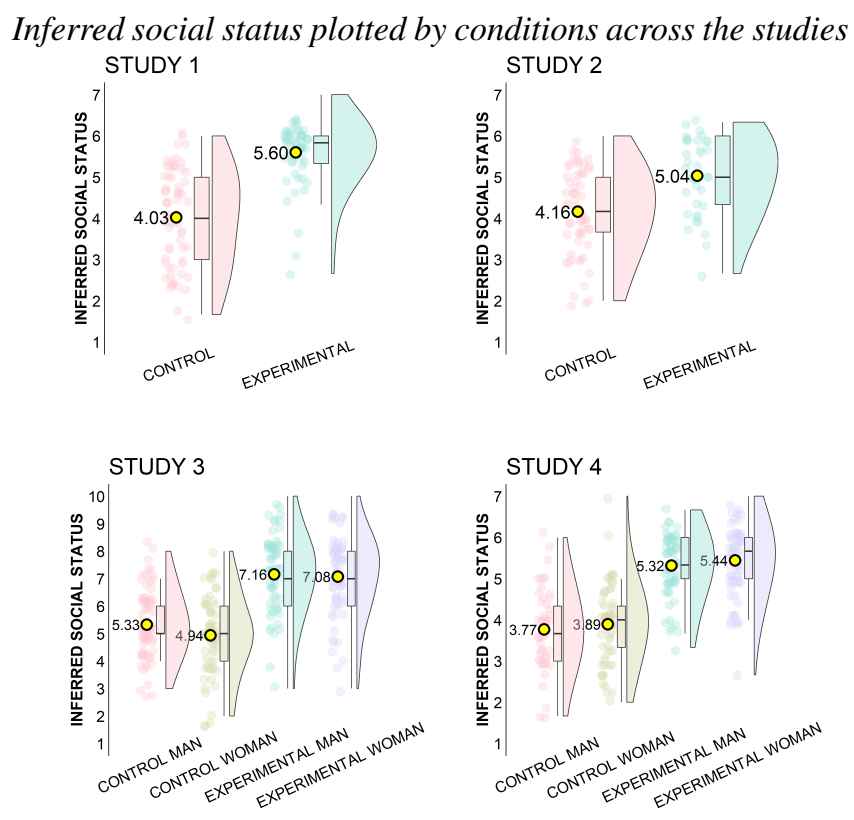

Note. The vertical lines in the centers of the boxplots indicate the medians, with the areas above and below the medians corresponding to the interquartile ranges. The whiskers around the boxplots extend over 1.5 times the interquartile ranges. The shaded areas on the right side of the boxplots indicate the distributions of the data points. The shaded dots on the left side of the boxplots represent individual data points, and the yellow dots indicate means whose numerical values are rounded to two decimal places next to the yellow dots.

Then, participants filled out three scales measuring perceived life stress, affect, and anxiety. We measured how often participants experienced stress in their lives on a 10item Perceived Stress Scale (Cohen \& Williamson, 1988), anchored at $0=$ Never and $4=$ Very often, with items averaged to create a perceived life stress index ( $\alpha=.90, M=$ $1.89, S D=0.72$ ). Anxiety was measured with a six-item short-form of the Spielberger State-Trait Anxiety Inventory (Marteau \& Bekker, 1992), anchored at $1=$ Not at all and 
$4=$ Very much. These items were also averaged to create an anxiety index $(\alpha=.90, M=2.16, S D=0.75)$. Positive and negative affect elicited by the social media posts was captured using the 20-item (10 for positive affect and 10 for negative affect) Positive and Negative Affect Schedule (Watson et al., 1988), anchored at $1=$ very slightly or not at all and $5=$ extremely. We created a negative $(\alpha=.91, M=$ $1.74, S D=0.77)$ and a positive $(\alpha=.91, M=2.85, S D=$ 0.82 ) affect index by averaging these items. The scale formats across these scales were altered to mitigate problems associated with common method bias (Gasiorowska et al., 2022; Podsakoff et al., 2003). Finally, participants provided demographic information.

\section{Results and Discussion}

\section{Comparisons between the two control conditions}

We began our analysis with a multivariate analysis of variance (MANOVA) to determine if there was a difference between the two control conditions in the measurements taken in this study. The analysis revealed no differences between the two control conditions, $F(6,55)=1.84$, Wilk's lamba $=$ $0.83, p=.108$. Therefore, we merged the two control conditions to allow for a parsimonious analysis (for a similar procedure, see Griskevicius et al., 2009; Griskevicius et al., 2010).

\section{Manipulation check}

A Welch two-sample $t$-test on the manipulation check index revealed that participants rated Alicia's beautification efforts as higher in the experimental condition than in the control condition $\left(M_{\text {experimental }}=5.59, S D=1.44 ; M_{\text {control }}=\right.$ 3.73, $S D=1.34), t(73,67)=-6.42, p<.001, d=1.35$.

\section{Inferred status}

A similar analysis revealed that participants rated Alicia's social status as higher in the experimental condition than in the control condition $\left(M_{\text {experimental }}=5.04, S D=0.94\right.$; $\left.M_{\text {control }}=4.16, S D=1.04\right), t(84.35)=-4.34, p<.001, d$ $=0.87$.

\section{Robustness tests}

To ensure that the experimental manipulation, rather than any of our potential confounds, caused the shifts in status ratings, we conducted an analysis of covariance (ANCOVA) with the experimental condition as the predictor and inferred social status as the dependent variable. We included the following covariates in the model: perceived life stress, affect, and anxiety indexes. Within this model, the main effect of the experimental condition remained statistically significant, $F(1,94)=19.13, \mathrm{p}<.001, \eta_{p}^{2}=.17$. However, none of our covariates were significantly associated with status inferences: perceived life stress: $F(1,94)=2.46, p=.120, \eta_{p}^{2}=$
.03 ; anxiety: $F(1,94)=0.03, p=.871, \eta_{p}^{2}<.01$; negative affect: $F(1,94)=0.06, p=.800 ; \eta_{p}^{2}<.01$; positive affect: $F(1,94)=0.01, p=.908, \eta_{p}^{2}<.01$.

\section{Discussion}

Study 2 provides evidence for the generalizability of our focal effect across samples and rules out several potential confounds, such as anxiety and negative affect. Crucially, we found no significant differences in the measurements in Study 2 between the two control conditions in which participants were either exposed to images of a fictional character or merely the social media logo of Instagram. Thus, the experimental manipulation rather than the choice of control stimuli emerges as the most plausible explanation underlying our results on inferred social status.

\section{Study 3}

Marketing activities and academic literature related to cosmetic procedures focus on women, but men are also increasingly interested in these practices (Sinno et al., 2016). Whereas Studies 1-2 found a strong effect of our manipulation on status inferences attributed to a female target, it remains unclear whether the same effect applies to men. We examine this possibility in Study 3, in which we conceptually replicate and extend the findings from Studies 1-2 with a different manipulation and another way of measuring the dependent variable (Crandall \& Sherman, 2016). This study was preregistered (https://aspredicted.org/645DQP).

\section{Participants and Procedure}

In line with the preregistered protocol, we recruited 266 participants (159 women, $M_{\text {age }}=36.6$ years, $S D=12.5$ ) from the United States.

This study used a 2 (target sex: man or woman) $\times 2$ (condition: control or experimental) between-subjects design. After participants accepted an informed consent form, they were assigned to one of the four conditions: the control condition, in which they rated the social status of a fictitious man $(n=73)$ or woman $(n=65)$, or the experimental condition, in which they also rated the social status of a fictitious man $(n=63)$ or woman $(n=65)$. Participants in the control condition read about a person going to a car inspection ("Andrew [Alicia] is 35 years old. His [her] car has not been inspected for a while. Therefore, today, he [she] is going for an annual, routine car check-up."). Participants in the experimental condition read about a person going for a medical check-up ("Andrew [Alicia] is 35 years old. He [she] has undergone a rhinoplasty (nose job) few weeks ago. Therefore, today, he [she] is going for a scheduled check-up with his [her] plastic surgeon."). Then, they were instructed to "Write three (3) sentences describing what happened during 
the check-up," followed by completing the three-item manipulation check measure as described in our previous studies, with participants' responses averaged to form a manipulation check index $(\alpha=.96, M=4.89, S D=1.48)$. In this study, we introduced a new measure of social status. Specifically, socioeconomic status (SES) was measured using the MacArthur Scale of Subjective Social Status (Adler et al., 2000), where participants had to rank themselves on a rug of the subjective social status ladder after reading the following instructions:

Think of this ladder as representing where people stand in the United States. At the top of the ladder are the people who are the best off, those who have the most money, most education, and the best jobs. At the bottom are the people who are the worst off, those who have the least money, least education, worst jobs, or no job. Place Andrew [Alicia] on the rung that best represents where you think he [she] stands on the ladder.

Participants responded on a 10-point scale anchored at 1 $=$ worst off and $10=$ best off $(M=6.09, S D=1.67)$. Such single-item scales are valid if they represent clear and unambiguous constructs, as in the current case (Bergkvist \& Rossiter, 2007; Otterbring, 2020). Finally, participants provided information on their demographics, including their annual income from $\$ 10,000$ and less to $\$ 100,000$ and more, with intervals of $\$ 1,000$.

\section{Results and Discussion}

\section{Manipulation check}

A two-way ANOVA performed on the manipulation check index revealed a main effect of condition, with participants rating the beautification efforts of the targets as higher in the experimental condition than in the control condition $\left(M_{\text {experimental }}=6.09, S D=0.88 ; M_{\text {control }}=3.78, S D=0.96\right)$, $F(1,262)=221.46, p<.001, \eta_{p}^{2}=.46$. We also found a main effect of target sex such that participants rated a fictional woman as more concerned with beautification practices than a fictional man $\left(M_{\text {woman }}=5.11, S D=1.45 ; M_{\text {man }}\right.$ $=4.68, S D=1.48), F(1,262)=5.46, p=.020, \eta_{p}^{2}=.02$. However, there was no significant interaction target sex $\times$ condition interaction, $F(1,262)=0.04, p=.851, \eta_{p}^{2}<.01$.

\section{Inferred status}

A similar analysis on the item capturing inferred social status revealed, consistent with our theorizing, a main effect of condition, such that participants in the experimental condition perceived the targets to be higher in status than their peers in the control condition $\left(M_{\text {experimental }}=7.12, S D=\right.$ $\left.1.40 ; M_{\text {control }}=5.14, S D=1.30\right), F(1,262)=62.74, p<$
$.001, \eta_{p}^{2}=.19$. However, we found no main effect of target $\operatorname{sex}\left(M_{\text {woman }}=6.01, S D=1.75 ; M_{\text {man }}=6.18, S D=1.58\right)$, $F(1,262)=2.90, p=.090, \eta_{p}^{2}=.01$, and no target sex $\times$ condition interaction, $F(1,262)=0.87, p=.351, \eta_{p}^{2}<.01$.

\section{Robustness tests}

To account for the possibility that our results were influenced by participants' income levels rather than our manipulation, we added participants' self-reported income-which is representative of their objective socioeconomic status-as a covariate to the model described above. Within this model, the main effect of condition remained significant, $F(1,261)$ $=63.19, p<.001, \eta_{p}^{2}=.19$. Further, we found a main effect of target sex such that the female target was seen as lower in social status than the male target, $F(1,261)=4.43, p=$ $.036, \eta_{p}^{2}=.02$. There was also a positive association between participants' self-reported income and their inferred social status of the fictional characters, $F(1,261)=9.01, p=.003$, $\eta_{p}^{2}=.03$. However, the target sex $\times$ condition interaction remained non-significant, $F(1,261)=1.15, p=.284, \eta_{p}^{2}<$ .01 .

\section{Discussion}

Study 3 provides preregistered evidence for notion that disclosing information about undergoing cosmetic procedures increases inferred social status, thus conceptually replicating the results from Studies 1-2 using a different way of capturing status inferences. We also demonstrate that the findings on status inferences generalize across sexes: both men and women derive more status benefits from disclosing information about having undergone cosmetic surgery compared to disclosing information about equally expensive daily activities.

\section{Study 4}

Whereas Studies 1-3 provide evidence for the generalizability and robustness of our results, it remains unknown what mechanism drives these findings. We propose that physical attractiveness may be a mediator of this effect. Inferred physical attractiveness has been studied as a potential status cue, with participants attributing higher (vs. lower) status to people on photographs depicting more (vs. less) attractive individuals (Kalick, 1988). However, Anderson et al. (2001) found that this effect was sex-specific; that is, physical attractiveness was positively related to status for men but not women (also see Krems et al., 2022). Therefore, in Study 4, we examine whether our status effect is driven by ratings of physical attractiveness and whether it is sex-specific or applies across the sexes. 


\section{Participants and Procedure}

We used the same approach to estimating sample size as in the previous study; therefore, we recruited 236 participants (114 women, $M_{\text {age }}=32.7$ years, $S D=9.2$ ) from the United States.

This study used a 2 (target sex: man or woman) $\times 2$ (condition: control or experimental) between-subject design. Participants first accepted an informed consent form and were then assigned to one of four conditions: the control condition, in which they saw a fictional man $(n=50)$ or woman $(n$ $=60)$, or the experimental condition, in which they were also exposed to a fictional man $(n=63)$ or woman $(n=63)$. We used the same manipulation here as in Study 3. Participants answered the three manipulation check items used across all prior studies and we averaged these items to create a manipulation check index $(\alpha=.95, M=4.88, S D=1.48)$. Similarly, the three items capturing the inferred status of the fictional character, as used in Studies 1-2, were averaged to create an index of social status ( $\alpha=.92, M=4.66, S D=1.17)$. Participants also rated the physical attractiveness of the target person ("How physically attractive is Alicia [Andrew]?") on a 101-point scale anchored at $0=$ Very unattractive and 100 $=$ Very attractive $(M=55.73, S D=15.38)$. In the end of the study, participants provided demographic information.

\section{Results and Discussion}

\section{Manipulation check}

A two-way ANOVA performed on the manipulation check index revealed a main effect of condition, such that participants in the experimental condition rated the beautification efforts of the target character as higher than participants in the control condition $\left(M_{\text {experimental }}=5.86, S D=1.00\right.$; $\left.M_{\text {control }}=3.75, S D=1.08\right), F(1,232)=127.39, p<.001$, $\eta_{p}^{2}=.35$. However, we found no main effect of target sex $\left(M_{\text {woman }}=4.91, S D=1.40 ; M_{\text {man }}=4.84, S D=1.56\right), F(1$, $232)=1.65, p=.201, \eta_{p}^{2}<.01$, and no target sex $\times$ condition interaction, $F(1,232)=0.39, p=.531, \eta_{p}^{2}<.01$.

\section{Inferred status}

A similar analysis on status inferences revealed a main effect of condition, such that participants in the experimental condition rated the social status of the fictional character as higher than participants in the control condition $\left(M_{\text {experimental }}\right.$ $\left.=5.38, S D=0.83 ; M_{\text {control }}=3.84, S D=0.94\right), F(1,232)=$ 85.44, $p<.001, \eta_{p}^{2}=.27$. Again, we found no main effect of target sex $\left(M_{\text {woman }}=4.69, S D=1.20 ; M_{\text {man }}=4.63, S D=\right.$ $1.15), F(1,232)=0.57, p=.452, \eta_{p}^{2}<.01$ and no target sex $\times$ condition interaction, $F(1,232)<.01, p=.997, \eta_{p}^{2}<.01$.

\section{Physical attractiveness}

Replacing the dependent variable with perceived physical attractiveness showed no significant main effect of condition $\left(M_{\text {experimental }}=52.82, S D=11.92 ; M_{\text {control }}=58.27, S D=\right.$ $17.52), F(1,232)=0.45, p=.503, \eta_{p}^{2}<.01$, and no main effect of target sex $\left(M_{\text {woman }}=59.14, S D=15.32 ; M_{\text {man }}=\right.$ 52.02, $S D=14.63), F(1,232)=1.44, p=.231, \eta_{p}^{2}<.01$. However, we found a marginally significant target sex $\times$ condition interaction, $F(1,232)=3.81, p=.052, \eta_{p}^{2}=.02$. We performed simple slopes analysis to understand the nature of this interaction and found that the slope of the experimental condition for male targets was not significant, $b=1.86,95 \%$ CI $[-3.64,7.37], t(111)=0.67, p=.504$, but it was significant and positive for female targets, such that participants in the experimental condition rated female targets as more physically attractive than participants in the control condition, $b=$ $9.35,95 \%$ CI $[4.12,14.58], t(121)=3.54, p<.001$.

\section{Moderated mediation}

To test whether the cosmetic surgery effect on participants' status inferences would be indirect through their attractiveness perceptions and whether this presumed interplay would be contingent on target sex, we conducted a moderated mediation analysis (PROCESS Model 7, Hayes, 2017). Condition $(0=$ control; $1=$ experimental $)$ was the predictor, participants' attractiveness perceptions (continuous) acted as the mediator, target sex ( $0=$ man; $1=$ woman $)$ served as the moderator, and participants' status inferences (continuous) acted as the outcome variable. The model explained roughly $50 \%$ of the variance in participants' status inferences and was significant, $F(2,233)=118.70, p<.001, R^{2}=.50$. More importantly, and consistent with our theorizing, a bootstrap procedure that generated a sample size of 5,000 revealed that the index of moderated mediation was statistically significant, as evidenced from the $95 \%$ confidence interval (CI), which did not contain zero $(95 \% \mathrm{CI}=[.002, .30])$. Specifically, the conditional indirect effect of the experimental (vs. control) condition on participants' status inferences through their attractiveness perceptions was significant for the female model $(95 \% \mathrm{CI}=[.08, .32])$ but was non-significant for the male model $(95 \% \mathrm{CI}=[-.07, .16])$. Controlling for participant sex as a covariate did not change the nature or significance of these results, and participant sex was not significantly associated with either attractiveness perceptions or status inferences.

\section{Discussion}

Study 4 replicates the results from our former studies by showing that disclosure of information about participation in cosmetic procedures led to increased inferences about social status. Moreover, we found this effect to be partially driven by an increased evaluation of the target's physical attractive- 
ness; however, this "beauty premium" only created positive spillover effects on status inferences for female but not male targets.

\section{Study 5}

Our results consistently show that people can increase their status by ostensibly disclosing involvement in cosmetic procedures. But does the desire to increase social status motivate people to engage in such practices? According to frameworks on psychological compensation, people who become deprived of attributes important to their self-view or identity strive to regain or replace those attributes, both cognitively and behaviorally (Bäckman \& Dixon, 1992; Mandel et al., 2017; Otterbring et al., 2022). If disclosure of information about undergoing cosmetic procedures is recognized as a status signal, then consumers should be more willing to undergo such treatments if their social rank is threatened (Otterbring et al., 2018; Rucker \& Galinsky, 2008; Sivanathan \& Pettit, 2010). We test this prediction experimentally in Study 5.

\section{Participants and Procedure}

We maximized the sample size considering our existing budget constraints and hence recruited 400 participants (195 women, $M_{\text {age }}=36.1$ years, $S D=13.0$ ) from the United States.

After participants agreed to an informed consent form, they were assigned to one of two between-subjects conditions in which we experimentally heightened $(n=196)$ or lowered $(n=204)$ their relative social rank. In the "heightened" social rank condition, participants compared themselves to individuals occupying the bottom rungs of the relative socioeconomic status ladder, whereas in the "lowered" social rank condition, participants compared themselves to others occupying the top rungs of the ladder (Adler et al., 2000):

Think of the ladder above as representing where people stand in the United States. Now, please compare yourself to the people at the very bottom [top] of the ladder. These are people who are the worst [best] off - those who have the least [most] money, least [most] education, and the least [most] respected jobs. In particular, we'd like you to think about how you are different from these people in terms of your own income, educational history, and job status. Where would you place yourself on this ladder relative to these people at the very bottom [top]?

To increase the likelihood that our manipulation would elicit desired feelings of relatively heightened or lowered social rank (for a similar procedure, see Piff et al., 2010), we then asked them to "Imagine yourself in getting acquainted interaction with one of the people you just thought about from the bottom [top] of the ladder above." Their task was to write two to five sentences describing such a possible interaction in terms of what participants would have talked about, how the interaction would have proceeded, etc.

Next, on a 201-point scale anchored at $-100=$ Very unlikely and $100=$ Very likely, participants indicated how willing they were to undergo cosmetic surgery in the future ("How likely would you be to undergo a cosmetic surgery at some stage of your life to look better?"), followed by them indicating their self-perceived physical attractiveness ("How physically attractive do you think you are?") on a 10-point scale anchored at $1=$ Not at all attractive and $10=$ Very attractive. Subsequently, participants completed a six-item measure of their status seeking motivation from the Fundamental Social Motives Inventory (Neel et al., 2016), with responses ranging from $1=$ strongly disagree, to $7=$ strongly agree. We created a status seeking index by averaging responses to these items ( $\alpha=.83, M=3.78, S D=1.21)$. Finally, participants provided their demographic data.

\section{Results and Discussion}

\section{Willingness to undergo cosmetic surgery}

A Welch two-sample $t$-test on the self-reported likelihood of undergoing cosmetic surgery found that participants whose relative social rank was lowered (vs. heightened) were more willing to undergo cosmetic surgery in the future $\left(M_{\text {lowered }}=-45.46, S D=63.64 ; M_{\text {hightened }}=-59.88, S D=\right.$ $55.89), t(394.85)=-2.41, p=.016, d=0.24(\text { see Figure } 2)^{1}$.

As a secondary test of our hypothesis, we fitted a linear model with willingness to undergo cosmetic surgery as the dependent variable and with the (measured) social status index as the predictor, controlling for the effect of the (manipulated) experimental assignment into different conditions. The results of this model showed a marginally significant effect of the status seeking index on willingness to undergo cosmetic surgery, $b=4.76,95 \%$ CI $[-0.10,9.62], t(397)=1.92, p$ $=.055$. Importantly, however, the effect of the experimental condition remained significant, $b=14.43,95 \%$ CI [2.68, 26.18], $t(397)=2.41, p=.016$.

\section{Robustness tests}

We conducted a a one-way ANCOVA to test whether our our focal effect was robust to the inclusion of self-assessed physical attractiveness and the (measured) status seeking index as covariates. After including these two covariates in

\footnotetext{
${ }^{1}$ As shown in Figure 2, the dependent variable was not normally distributed. Therefore, we supplemented our main analysis with the Wilcoxon rank sum test, which indeed confirmed a difference between conditions in the propensity to undergo cosmetic surgery, $p=.009$.
} 
the model, the main effect our (manipulated) social rank factor remained significant, $F(1,397)=5.75, p=.017, \eta_{p}^{2}=$ .01. Further, we found a main effect of the (measured) status seeking index, such that the more status seeking were participants, the more inclined they were to undergo cosmetic surgery, $F(1,396)=3.87, p=.050, \eta_{p}^{2}<.01$. Selfassessment of physical attractiveness was not predictive of willingness to undergo cosmetic surgery, $F(1,397)<.01, p$ $=.939, \eta_{p}^{2}<.01$.

\section{Figure 2}

Willingness to undergo cosmetic surgery



Note. The black dots in the boxplots indicate the means, and their 95\% bootstrapped confidence intervals are represented by the black lines above and below, with the numerical means rounded to one decimal place next to these dots. The boxplot covers the data range between the first and third quartiles, with whiskers covering 1.5 times the interquartile ranges. The violin plots show the density of data points. The figure was truncated for readability.

\section{Discussion}

The results of Study 5 demonstrate that participants whose relative social rank is lowered (vs. heightened) are more motivated to undergo cosmetic procedures. This pattern of results also emerged among participants with a stronger status seeking motivation, thus indicating that both measured and manipulated status facets are positively associated with an increased willingness to engage in cosmetic surgery. Crucially, these effects remained significant after controlling for self-assessed physical attractiveness, which was not related to the responses on our focal dependent variable.

\section{General Discussion}

The current research provides empirical evidence for the status-signaling function of disclosing information about un- dergoing cosmetic surgery among both men and women. This is noteworthy given the widespread belief among surgeons and laypeople that patients derive aesthetic but not status-related benefits from cosmetic surgery. One surgeon we interviewed claimed that almost everyone can afford cosmetic procedures because of easy access to consumer credit, and our pilot study showed that people generally do not view such procedures as unusual or reserved only for people with a high social standing. Therefore, these procedures were not assumed to amplify status inferences. We contested this assumption and found that individuals who disclosed information about being cosmetic surgery patients were deemed as higher in status than individuals who disclosed information about everyday activities, even when such daily activities are equally expensive as cosmetic procedures. In addition, perceived attractiveness accounted for a substantial amount of the variance in status ratings for women but not for men. These results suggest that - in the case of womenstatus-related benefits of disclosing information about cosmetic surgery are partially explained by increases in perceived physical attractiveness.

Our final study showed that increasing the desire to attain status by experimentally eliciting feelings of relatively low (vs. high) status increased the desire for cosmetic surgery. Our findings are consistent with those of Xygalatas et al. (2021), who found that low-status Tamil Hindus are more likely to engage in physically costly rituals such as body modification through piercings than their high-status counterparts. The results reported herein also provide casual support for the previously established association between status striving and interest in cosmetic procedures found in US states with higher (vs. lower) income inequality, where status competition should be higher (X. Wang et al., 2021; see also Walasek et al., 2018; Walasek and Brown, 2015).

The existing literature on decision making related to cosmetic surgery has focused almost exclusively on women (e.g., Gimlin, 2000), although the number of cosmetic procedures performed on men has recently increased substantially (Sinno et al., 2016). We add to this literature by examining whether disclosure of information about cosmetic surgery has an impact on status inferences and perceptions of attractiveness in both men and women. In a mating context, men tend to benefit more than women from high status and women tend to benefit more than men from physical attractiveness (Buss, 1989; Li et al., 2002; Otterbring, 2018; Walter et al., 2020). Thus, it was reasonable to expect sexspecific effects of our manipulation (cosmetic surgery vs. everyday activities) on status inferences and perceptions of attractiveness. Nevertheless, we found that being informed that one had undergone cosmetic surgery increased status ratings across the sexes. However, in addition to such a status boost, women but not men received a "beauty premium" regarding perceptions of their attractiveness. 
Our findings that laypeople believe that cosmetic surgery provides mainly aesthetic benefits are consistent with previous literature based on interviews with patients and surgeons (e.g., Dorneles de Andrade, 2010). Because we found that participants viewed cosmetic surgery as a form of luxury consumption, sharing information about these procedures may likely elicit social perceptions associated with other forms of luxury consumption. People benefit from status signaling through luxury consumption by increasing compliance, labor market prospects, the amount of donations received, endowments in economic games, and mating opportunities without altering perceptions of their attractiveness (Nelissen \& Meijers, 2011; Sundie et al., 2011; Y. Wang \& Griskevicius, 2014). However, people who seek to signal status through luxury consumption sometimes face negative consequences. Status displays through such consumption can make an actor appear colder, less loyal, and immature, and can negatively affect perceptions of intelligence and friendliness (Cannon \& Rucker, 2019; Hudders et al., 2014). Therefore, consumers should be aware of these potentially negative consequences of conspicuous status displays. More importantly, in private shopping contexts, the experience of luxury consumption (vs. non-luxury consumption) may cause them to become more selfish, sharing fewer resources and donating less to charity, with this effect reversed in public settings (Y. Wang et al., 2021).

\section{Limitations and Future Directions}

Our theorizing assumes that the pursuit of status leads consumers to engage in behaviors that are physically and financially costly; however, it is also plausible that our results were at least partially confounded by differences in participants' use of impression management tactics. For example, people with higher levels of self-monitoring - the ability to adapt self-presentation strategies to social needs-are more likely to convey a positive image of themselves to others than people with lower levels of self-monitoring (Turnley \& Bolino, 2001). Thus, it is possible that we obtained relatively low effect sizes in Study 5 not because these effects are weak, but because the propensity to undergo cosmetic surgery to signal status was stronger or present only in individuals with high self-monitoring, while absent in individuals with low self-monitoring, who should be less sensitive to social cues. Further research should measure participants' propensity to engage in impression management activities.

There are two main ways to achieve high status: dominance, which involves the use of fear and intimidation to wield influence, and prestige, which is achieved by sharing skills, knowledge, and resources that are valued by a group (Cheng et al., 2013; Panchal \& Gill, 2020). Because we did not capture how participants perceived the target's dominance or prestige, it remains unknown whether a person who announces his or her involvement in cosmetic procedures is viewed as a more dominant or a more prestigious leader than a person who discloses information about more common activities (Van Vugt \& Smith, 2019). Future research should address this topic.

Whether people ascribe someone high status depends on the context. For example, a professor who is recognized in the academic community may be ascribed lower status in medical schools than a less renowned but practicing physician among medical students because they view the latter person's knowledge as more relevant to their careers (Barkow, 2014). Although we controlled for variables such as income levels (Study 3) and self-assessed physical attractiveness (Study 5), it is unclear whether the status-signaling function of disclosing involvement in cosmetic surgery is equally strong across social classes, cultures, and contexts. Such potential moderators are yet to be explored in future academic work.

Finally, recent research shows that people conspicuously display their health-related behaviors, such as exercise, in a mating state of mind (Kowal et al., 2022; Vandenbroele et al., 2020). Whereas we focused our study on the associations between conspicuous display of involvement in cosmetic procedures, it is also plausible that people who are trying to attract potential mates may be more willing to disclose their involvement in cosmetic procedures. Further research could test this possibility.

\section{References}

Adler, N. E., Epel, E. S., Castellazzo, G., \& Ickovics, J. R. (2000). Relationship of subjective and objective social status with psychological and physiological functioning: Preliminary data in healthy, white women. Health Psychology, 19(6), 586-592. https: //doi.org/10.1037/0278-6133.19.6.586

Anderson, C., Hildreth, J. A. D., \& Howland, L. (2015). Is the desire for status a fundamental human motive? A review of the empirical literature. Psychological Bulletin, 141(3), 574-601. https://doi.org/10.1037/ a0038781

Anderson, C., John, O. P., Keltner, D., \& Kring, A. M. (2001). Who attains social status? Effects of personality and physical attractiveness in social groups. Journal of Personality and Social Psychology, 81(1), 116-132. https://doi.org/10.1037/00223514.81 .1 .116

Bäckman, L., \& Dixon, R. A. (1992). Psychological compensation: A theoretical framework. Psychological Bulletin, 112(2), 259-283. https://doi.org/10.1037/ 0033-2909.112.2.259

Barkow, J. H. (2014). Prestige and the ongoing process of culture revision. In J. T. Cheng, J. L. Tracy, \& C. Anderson (Eds.), The psychology of social status 
(pp. 29-45). Springer. https://doi.org/10.1007/9781-4939-0867-7_2

Bellezza, S., Paharia, N., \& Keinan, A. (2017). Conspicuous consumption of time: When busyness and lack of leisure time become a status symbol. Journal of Consumer Research, 44(1), 118-138. https:// doi. org/10.1093/jcr/ucw076

Berger, J., Cohen, B. P., \& Zelditch Jr, M. (1972). Status characteristics and social interaction. American Sociological Review, 241-255. https:// doi org / 10. 2307/2093465

Bergkvist, L., \& Rossiter, J. R. (2007). The predictive validity of multiple-item versus single-item measures of the same constructs. Journal of Marketing Research, 44(2), 175-184. https://doi.org/10.1509/ jmkr.44.2.175

Bolker, B. (2007). Stochastic simulation and power analysis. http://citeseerx.ist.psu.edu/viewdoc/summary?doi= 10.1.1.720.8252

Branford, O. A., Kamali, P., Rohrich, R. J., Song, D. H., Mallucci, P., Liu, D. Z., Lang, D., Sun, K., Stubican, M., \& Lin, S. J. (2016). \# Plasticsurgery. Plastic and Reconstructive Surgery, 138(6), 1354-1365. https: //doi.org/10.1097/PRS.0000000000002814

Buss, D. M. (1989). Sex differences in human mate preferences: Evolutionary hypotheses tested in 37 cultures. Behavioral and Brain Sciences, 12(1), 1-14. https://doi.org/10.1017/S0140525X00023992

Cannon, C., \& Rucker, D. D. (2019). The dark side of luxury: Social costs of luxury consumption. Personality and Social Psychology Bulletin, 45(5), 767-779. https: //doi.org/10.1177/0146167218796790

Cheng, J. T., Tracy, J. L., Foulsham, T., Kingstone, A., \& Henrich, J. (2013). Two ways to the top: Evidence that dominance and prestige are distinct yet viable avenues to social rank and influence. Journal of Personality and Social Psychology, 104(1), 103-125. https://doi.org/10.1037/a0030398

Cohen, S., \& Williamson, G. (1988). Perceived stress in a probability sample of the United States. In $\mathrm{S}$. Shirlynn \& S. Oskamp (Eds.), The Social Psychology of Health: The Claremont Symposium on Applied Social Psychology (pp. 31-67). Sage.

Crandall, C. S., \& Sherman, J. W. (2016). On the scientific superiority of conceptual replications for scientific progress. Journal of Experimental Social Psychology, 66, 93-99. https://doi.org/10.1016/j.jesp.2015. 10.002

Dorneles de Andrade, D. (2010). On norms and bodies: Findings from field research on cosmetic surgery in Rio de Janeiro, Brazil. Reproductive Health Matters, 18(35), 74-83. https:// doi .org/10 . 1016/S09688080(10)35519-4
Driskell, J. E., \& Mullen, B. (1990). Status, expectations, and behavior: A meta-analytic review and test of the theory. Personality and Social Psychology Bulletin, 16(3), 541-553. https:// doi .org/10.1177/ 0146167290163012

Durante, K. M., \& Griskevicius, V. (2018). Evolution and consumer psychology. Consumer Psychology Review, 1(1), 4-21. https://doi.org/10.1002/arcp.1001

Fessler, D. M., Tiokhin, L. B., Holbrook, C., Gervais, M. M., \& Snyder, J. K. (2014). Foundations of the crazy bastard hypothesis: Nonviolent physical risk-taking enhances conceptualized formidability. Evolution and Human Behavior, 35(1), 26-33. https:// doi . org/10.1016/j.evolhumbehav.2013.09.003

Gasiorowska, A., Folwarczny, M., \& Otterbring, T. (2022). Anxious and status signaling: Examining the link between attachment style and status consumption and the mediating role of materialistic values. Personality and Individual Differences, 190, 111503. https://doi.org/10.1016/j.paid.2022.111503

Gierl, H., \& Huettl, V. (2010). Are scarce products always more attractive? The interaction of different types of scarcity signals with products' suitability for conspicuous consumption. International Journal of Research in Marketing, 27(3), 225-235. https:// doi. org/10.1016/j.ijresmar.2010.02.002

Gimlin, D. (2000). Cosmetic surgery: Beauty as commodity. Qualitative Sociology, 23(1), 77-98. https://doi.org/ 10.1023/A:1005455600571

Grand View Research. (2022). Cosmetic surgery and procedure market worth $\$ 145.7$ billion by 2030. https:// www.grandviewresearch.com/press-release/globalcosmetic-surgery-procedure-market\#

Griskevicius, V., Goldstein, N. J., Mortensen, C. R., Sundie, J. M., Cialdini, R. B., \& Kenrick, D. T. (2009). Fear and loving in Las Vegas: Evolution, emotion, and persuasion. Journal of Marketing Research, 46(3), 384-395. https://doi.org/10.1509/jmkr.46.3.384

Griskevicius, V., \& Kenrick, D. T. (2013). Fundamental motives: How evolutionary needs influence consumer behavior. Journal of Consumer Psychology, 23(3), 372-386. https://doi.org/10.1016/j.jcps.2013.03. 003

Griskevicius, V., Tybur, J. M., Sundie, J. M., Cialdini, R. B., Miller, G. F., \& Kenrick, D. T. (2007). Blatant benevolence and conspicuous consumption: When romantic motives elicit strategic costly signals. Journal of Personality and Social Psychology, 93(1), 85-102. https://doi.org/10.1037/0022-3514. 93.1 .85

Griskevicius, V., Tybur, J. M., \& Van den Bergh, B. (2010). Going green to be seen: Status, reputation, and conspicuous conservation. Journal of Personality and 
Social Psychology, 98(3), 392-404. https://doi.org/ 10.1037/a0017346

Hayes, A. F. (2017). Introduction to mediation, moderation, and conditional process analysis: A regressionbased approach. Guilford publications.

Heo, S., Moser, D. K., Chung, M. L., \& Lennie, T. A. (2012). Social status, health-related quality of life, and event-free survival in patients with heart failure. European Journal of Cardiovascular Nursing, 11(2), 141-149. https://doi.org/10.1016/j.ejcnurse. 2010.10 .003

Hudders, L., De Backer, C., Fisher, M., \& Vyncke, P. (2014). The rival wears Prada: Luxury consumption as a female competition strategy. Evolutionary Psychology, 12(3), 570-587. https:// doi . org/10 . 1177/ 147470491401200306

Ivanic, A. S., Overbeck, J. R., \& Nunes, J. C. (2011). Status, race, and money: The impact of racial hierarchy on willingness to pay. Psychological Science, 22(12), 1557-1566. https : // doi . org / 10 . 1177 / 0956797611419519

Kalick, S. M. (1988). Physical attractiveness as a status cue. Journal of Experimental Social Psychology, 24(6), 469-489. https://doi.org/10.1016/0022-1031(88) 90047-9

Kenrick, D. T., Saad, G., \& Griskevicius, V. (2013). Evolutionary consumer psychology: Ask not what you can do for biology, but. . Journal of Consumer Psychology, 23(3), 404-409. https://doi.org/10.1016/j. jcps.2013.04.003

Kowal, M., Sorokowski, P., Pisanski, K., Valentova, J. V., Varella, M. A., Frederick, D. A., Al-Shawaf, L., Garciéa, F. E., Giammusso, I., Gjoneska, B., et al. (2022). Predictors of enhancing human physical attractiveness: Data from 93 countries. Evolution and Human Behavior. https :// doi .org/10.1016/j. evolhumbehav.2022.08.003

Krems, J. A., Merrie, L. A., Short, V., Duarte, K., Rodriguez, N. N., French, J. E., Sznycer, D., \& Byrd-Craven, J. (2022). Third-party perceptions of male and female status: Male physical strength and female physical attractiveness cue high status. Frontiers in Ecology and Evolution, 294. https://doi.org/10.3389/fevo. 2022.860797

Li, N. P., Bailey, J. M., Kenrick, D. T., \& Linsenmeier, J. A. (2002). The necessities and luxuries of mate preferences: Testing the tradeoffs. Journal of Personality and Social Psychology, 82(6), 947. https://doi.org/ 10.1037//0022-3514.82.6.947

Mandel, N., Rucker, D. D., Levav, J., \& Galinsky, A. D. (2017). The compensatory consumer behavior model: How self-discrepancies drive consumer behavior. Journal of Consumer Psychology, 27(1),
133-146. https://doi.org/10.1016/j.jcps.2016.05. 003

Marteau, T. M., \& Bekker, H. (1992). The development of a six-item short-form of the state scale of the Spielberger State-Trait Anxiety Inventory (STAI). British Journal of Clinical Psychology, 31(3), 301306. https://doi.org/10.1111/j.2044-8260.1992. tb00997.x

Mofid, M. M., Teitelbaum, S., Suissa, D., RamirezMontañana, A., Astarita, D. C., Mendieta, C., \& Singer, R. (2017). Report on mortality from gluteal fat grafting: Recommendations from the ASERF Task Force. Aesthetic Surgery Journal, 37(7), 796806. https://doi.org/10.1093/asj/sjx004

Neel, R., Kenrick, D. T., White, A. E., \& Neuberg, S. L. (2016). Individual differences in fundamental social motives. Journal of Personality and Social Psychology, 110(6), 887-907. https:// doi .org/10.1037/ pspp0000068

Nelissen, R. M., \& Meijers, M. H. (2011). Social benefits of luxury brands as costly signals of wealth and status. Evolution and Human Behavior, 32(5), 343-355. https:// doi . org/10 .1016/j. evolhumbehav. 2010. 12.002

Nunes, J. C., Drèze, X., \& Han, Y. J. (2011). Conspicuous consumption in a recession: Toning it down or turning it up? Journal of Consumer Psychology, 21(2), 199-205. https://doi.org/10.1016/j.jcps.2010.11. 002

Otterbring, T. (2018). Healthy or wealthy? Attractive individuals induce sex-specific food preferences. Food Quality and Preference, 70, 11-20. https://doi.org/ 10.1016/j.foodqual.2017.02.014

Otterbring, T. (2020). Appetite for destruction: Counterintuitive effects of attractive faces on people's food choices. Psychology \& Marketing, 37(11), 14511464. https://doi.org/10.1002/mar.21257

Otterbring, T. (2021). Evolutionary psychology in marketing: Deep, debated, but fancier with fieldwork. Psychology $\mathcal{E}$ Marketing, 38(2), 229-238. https://doi.org/ 10.1002/mar.21453

Otterbring, T., Ringler, C., Sirianni, N. J., \& Gustafsson, A. (2018). The Abercrombie \& Fitch effect: The impact of physical dominance on male customers' status-signaling consumption. Journal of Marketing Research, 55(1), 69-79. https://doi.org/10.1509/ jmr. 15.0247

Otterbring, T., Samuelsson, P., Arsenovic, J., Truelsen, C. E., \& Folwarczny, M. (2022). Shortsighted sales or long-lasting loyalty? The impact of salespersoncustomer proximity on consumer responses and the beauty of bodily boundaries. In press, European Journal of Marketing. 
Otterbring, T., Sundie, J., Li, Y. J., \& Hill, S. (2020). Evolutionary psychological consumer research: Bold, bright, but better with behavior. Journal of Business Research, 120, 473-484. https://doi.org/10.1016/j. jbusres.2020.07.010

Panchal, S., \& Gill, T. (2020). When size does matter: Dominance versus prestige based status signaling. Journal of Business Research, 120, 539-550. https://doi. org/10.1016/j.jbusres.2019.03.047

Piff, P. K., Kraus, M. W., Côté, S., Cheng, B. H., \& Keltner, D. (2010). Having less, giving more: The influence of social class on prosocial behavior. Journal of Personality and Social Psychology, 99(5), 771784. https://doi.org/10.1037/a0020092

Podsakoff, P. M., MacKenzie, S. B., Lee, J.-Y., \& Podsakoff, N. P. (2003). Common method biases in behavioral research: A critical review of the literature and recommended remedies. Journal of Applied Psychology, 88(5), 879-903.

Rucker, D. D., \& Galinsky, A. D. (2008). Desire to acquire: Powerlessness and compensatory consumption. Journal of Consumer Research, 35(2), 257267. https://doi.org/10.1086/588569

Saad, G. (2013). Evolutionary consumption. Journal of Consumer Psychology, 23(3), 351-371. https://doi.org/ 10.1016/j.jcps.2013.03.002

Saad, G., \& Vongas, J. G. (2009). The effect of conspicuous consumption on men's testosterone levels. Organizational Behavior and Human Decision Processes, 110(2), 80-92. https://doi.org/10.1016/j.obhdp. 2009.06.001

Schaller, M., Kenrick, D. T., Neel, R., \& Neuberg, S. L. (2017). Evolution and human motivation: A fundamental motives framework. Social and Personality Psychology Compass, 11(6), e12319. https://doi . org/10.1111/spc3.12319

Sinno, S., Lam, G., Brownstone, N. D., \& Steinbrech, D. S. (2016). An assessment of gender differences in plastic surgery patient education and information in the United States: Are we neglecting our male patients? Aesthetic Surgery Journal, 36(1), 107-110. https://doi.org/10.1093/asj/sjv100

Sivanathan, N., \& Pettit, N. C. (2010). Protecting the self through consumption: Status goods as affirmational commodities. Journal of Experimental Social Psychology, 46(3), 564-570. https://doi.org/10.1016/j. jesp.2010.01.006

Stoet, G. (2010). PsyToolkit: A software package for programming psychological experiments using linux. Behavior Research Methods, 42(4), 1096-1104. https://doi.org/10.3758/BRM.42.4.1096

Stoet, G. (2017). PsyToolkit: A novel web-based method for running online questionnaires and reaction-time ex- periments. Teaching of Psychology, 44(1), 24-31. https://doi.org/10.1177/0098628316677643

Sundie, J. M., Kenrick, D. T., Griskevicius, V., Tybur, J. M., Vohs, K. D., \& Beal, D. J. (2011). Peacocks, Porsches, and Thorstein Veblen: Conspicuous consumption as a sexual signaling system. Journal of Personality and Social Psychology, 100(4), 664680. https://doi.org/10.1037/a0021669

Turnley, W. H., \& Bolino, M. C. (2001). Achieving desired images while avoiding undesired images: Exploring the role of self-monitoring in impression management. Journal of Applied Psychology, 86(2), 351360. https://doi.org/10.1037/0021-9010.86.2.351

Van Vugt, M., \& Smith, J. E. (2019). A dual model of leadership and hierarchy: Evolutionary synthesis. Trends in Cognitive Sciences, 23(11), 952-967. https://doi. org/10.1016/j.tics.2019.09.004

Vandenbroele, J., Van Kerckhove, A., \& Geuens, M. (2020). If you work it, flaunt it: Conspicuous displays of exercise efforts increase mate value. Journal of Business Research, 120, 586-598. https://doi.org/10. 1016/j.jbusres.2019.01.030

Veblen, T. (1899). The theory of the leisure class. Macmillan.

Walasek, L., Bhatia, S., \& Brown, G. D. (2018). Positional goods and the social rank hypothesis: Income inequality affects online chatter about high-and lowstatus brands on twitter. Journal of Consumer Psychology, 28(1), 138-148. https://doi.org/10.1002/ jcpy. 1012

Walasek, L., \& Brown, G. D. (2015). Income inequality and status seeking: Searching for positional goods in unequal us states. Psychological Science, 26(4), 527533. https://doi.org/10.1177/0956797614567511

Walter, K. V., Conroy-Beam, D., Buss, D. M., Asao, K., Sorokowska, A., Sorokowski, P., Aavik, T., Akello, G., Alhabahba, M. M., Alm, C., et al. (2020). Sex differences in mate preferences across 45 countries: A large-scale replication. Psychological Science, 31(4), 408-423. https:// doi.org/10.1177/ 0956797620904154

Wang, J., \& Yu, Y. (2022). Beautify the blurry self: Low selfconcept clarity increases appearance management. Journal of Consumer Psychology, 1-17. https://doi. org/10.1002/jcpy. 1298

Wang, X., Chen, H., Chen, Z., \& Yang, Y. (2021). Women's intrasexual competition results in beautification. Social Psychological and Personality Science, 12(5), 648-657. https:// doi.org/10.1177/ 1948550620933403

Wang, Y., \& Griskevicius, V. (2014). Conspicuous consumption, relationships, and rivals: Women's luxury products as signals to other women. Journal of 
Consumer Research, 40(5), 834-854. https:// doi. org/10.1086/673256

Wang, Y., John, D. R., \& Griskevicious, V. (2021). Does the devil wear Prada? Luxury product experiences can affect prosocial behavior. International Journal of Research in Marketing, 38(1), 104-119. https://doi. org/10.1016/j.ijresmar.2020.04.001

Watson, D., Clark, L. A., \& Tellegen, A. (1988). Development and validation of brief measures of positive and negative affect: The PANAS Scales. Journal of Personality and Social Psychology, 54(6), 10631070.
Xygalatas, D., Maňo, P., Bahna, V., Klocová, E. K., Kundt, R., Lang, M., \& Shaver, J. H. (2021). Social inequality and signaling in a costly ritual. Evolution and Human Behavior, 42(6), 524-533. https://doi. org/10.1016/j.evolhumbehav.2021.05.006

Zahavi, A. (1975). Mate selection-A selection for a handicap. Journal of Theoretical Biology, 53(1), 205214. https://doi.org/10.1016/0022-5193(75)901113 